\title{
MÉTODOS TOPSIS E VIKOR NA VERIFICAÇÃO DA RESOURCE ADVANTAGE THEORY EM EMPRESAS TÊXTEIS BRASILEIRAS
}

\author{
Moacir Manoel Rodrigues Junior \\ moacir_ro@hotmail.com \\ Fernanda Kreuzberg \\ Universidade Regional de Blumenau \\ fernandakreuzberg@gmail.com \\ Marlon Jeske \\ Universidade Regional de Blumenau \\ marlon_jeske@hotmail.com \\ Flávio Rodrigues \\ Universidade Regional de Blumenau \\ rodrigues.flavio96@gmail.com
}

Universidade Federal do Paraná/Universidade Regional de Blumenau

\begin{abstract}
Resumo
Esta pesquisa teve o objetivo analisar o desempenho em recursos, mercado e financeiro que explicam a vantagem competitiva empresarial de acordo com a Resource Advantage Theory (RA Theory), de empresas do setor têxtil listadas na BM\&FBovespa. Foram analisadas um total de 21 empresas do setor têxtil divididas em quatro segmentos. Para a mensuração do desempenho foram adotados os métodos de apoio a decisão multicritério baseados em cenários ideais TOPSIS e VIKOR. Foram analisados os desempenhos com base em quatro indicadores para recursos, três para mercado e quatro para a dimensão financeira. Os resultados da pesquisa apontam para a não verificação da RA Theory em empresas têxteis, considerando o que se preconiza de que uma empresa em vantagem em recursos e mercado tende a ser superior na dimensão financeira. Desta forma a única empresa que verificou este fator foi a Arezzo. De mesmo modo para verificação da referida teoria seria coerente que existisse correlação entre os rankings das diferentes dimensões, o que não se verificou. Assim conclui-se que a vantagem competitiva em recursos e em mercado de empresas do setor têxtil do Brasil não contribui para superioridade no desempenho financeiro das empresas.

Palavras-Chave: Resource Advantage Theory. Apoio a Decisão Multicritério. Indústrias Têxteis.
\end{abstract}

\begin{abstract}
This research had the objective to analyze the performance in Resources, Market and Financial explaining the competitive advantage of companies according to the Resource Advantage Theory, of Textile companies sector listed at BM\&FBovespa. It analyzed 21 companies in the textile sector divided into four segments. For measuring performance have been adopted the Multiple Criteria Decision Making based on ideal scenarios, TOPSIS and VIKOR. It was analyzed the performances based on four indicators for resources, three for market and four for the financial dimension. The results point to the non-verification of RA Theory in textile companies, considering what it recommends that a business advantage in resources and market tends to be higher in the financial dimension. In this way, the only company that observed this factor was the Arezzo. Likewise for verification of such a theory would be consistent that there was correlation between the rankings of different dimensions, which was not the case. It was concluded that competitive advantage in resources and in market
\end{abstract}


of companies, Brazil's textile sector does not contribute to high performance in financial dimension.

Key-words: $\quad$ Resource Advantage Theory. Multiple Criteria Decision Making. Textile Industry.

\section{INTRODUÇÃO}

Na década de 90, Hunt e Morgan (1995) publicaram um trabalho acerca das vantagens comparativas de recursos para as organizações. No período posterior os autores publicaram outro trabalho, inserindo em sua abordagem o desempenho de recursos, financeiro e de mercado das organizações como uma maneira de alcançar a vantagem competitiva. Esse trabalho de Hunt e Morgan (1996) culminou no desenvolvimento da Resource Advantage Theory (RA Theory), que conforme Rossi e Silva (2009) contestava algumas premissas da Economia Neoclássica.

Considerando a abordagem da RA Theory a competição baseia-se na disputa das empresas por vantagens comparativas de recursos que acarretam em vantagens competitivas, bem como em um desempenho superior no mercado (HUNT, 2000, 1997). Rossi e Silva (2009) salientam que essa abordagem pode ser classificada como de relações causa e efeito. Pois a vantagem competitiva de uma empresa frente as demais do mercado é uma consequência das vantagens comparativas de recursos, que causa um desempenho financeiro superior.

Dessa forma, mediante a RA Theory, Hunt (1997) salienta que as empresas estão inseridas em um processo competitivo objetivando sempre alcançar vantagens sobre as demais empresas. Assim, as empresas competem nas dimensões de recursos, mercado e performance financeira, considerando que as empresas com melhores vantagens de recursos possuem melhor posicionamento no mercado e melhor performance financeira (HUNT; MORGAN, 1996). Em virtude disso, aumenta-se a vantagem competitiva, pois as empresas buscam alcançar sempre um desempenho superior as demais, investindo assim em inovação para incrementar seus resultados (HUNT, 2000).

Diante disso, assume-se a seguinte problemática de pesquisa: $\mathrm{O}$ desempenho de recursos, mercado e financeiro explica a vantagem competitiva empresarial conforme a premissa da Resource Advantage Theory nas empresas do setor têxtil listadas na BM\&FBovespa? Para responder a problemática proposta, a pesquisa tem por objetivo analisar o desempenho de recursos, mercado e financeiro que explicam a vantagem competitiva empresarial de acordo com a Resource Advantage Theory, de empresas do setor têxtil listadas na BM\&FBovespa.

A justificativa para a restrição da aplicação do estudo com as empresas do setor têxtil, baseia-se em Slack (1993), considerando que este setor atende as todas as dimensões propostas pela Resource Advantage Theory, bem como se relaciona com a vantagem competitiva das empresas.

As contribuições desta pesquisa, prevalecem principalmente quanto aos procedimentos metodológicos. Pois efetua-se a mensuração do desempenho das três dimensões (recursos, mercado e financeiro) e efetua-se a construção de rankings para estabelecer a o posicionamento das empresas nessas dimensões da RA Theory, para assim explicar a vantagem competitiva. A construção dos rankings baseia-se em dois modelos distintos de apoio a tomada de decisão que envolvem a comparação de cenários (TOPSIS e VIKOR). A escolha desses dois métodos baseia-se na sua pouca aplicabilidade em estudos nacionais e também na sua operacionalização sujeito a pouca subjetividade.

Os métodos Technique for Order Preference by Similarity to an Ideal Solution (TOPSIS) proposto por Hwang e Yoon (1981) e VlseKriterijumska Optimizacija I Kompromisno Resenje (VIKOR) proposto por Opricovic (1998) veem sendo muito aplicados a pesquisas científicas voltadas ao apoio a decisão multicritério. Estes métodos objetivam estabelecer um ranking com base nas distâncias para como um cenário ideal. 
A diferença destes dois métodos vincula-se as funções de compromisso. No estudo de Opricovic e Tzeng (2004) tem-se uma discussão acerca das diferenças entre os métodos TOPSIS e VIKOR. Conforme os autores o método VIKOR ponderar a importância das distâncias em relação aos cenários Ideal e melhor desempenho verificado. Para os autores, a formação de rankings pelo método VIKOR torna-se mais estável se comparado ao método TOPSIS.

\section{REFERENCIAL TEÓRICO}

Nessa seção apresentam-se os principais aspectos teóricos da Resource Advantage Theory. Na sequência apresenta-se a descrição dos métodos TOPSIS e VIKOR, utilizados para mensurar o desempenho de recursos, mercado e financeiro das empresas que explicam a sua vantagem competitiva.

\subsection{RESOURCE ADVANTAGE THEORY}

A discussão em torno do surgimento da Resource Advantage Theory (RA Theory) iniciou na década de 90 com os trabalhos de Hunt e Morgan (1995, 1996). Naquele momento os autores inseriram a abordagem da vantagem competitiva por meio do desempenho de recursos, de mercado e performance financeira. Conforme Hunt (2000) as empresas estão em luta constante por alcançar vantagens de recursos e consequentes reflexos no mercado ao qual estão inseridas.

A competição no desempenho de recursos, nas posições de mercado e a performance financeira das empresas são o cerne da RA Theory, conforme esquematizado na Figura 1. Dessa forma, Hunt e Morgan (1996) consideram que quanto maior a vantagem de recursos, melhor o desempenho financeiro e de mercado.

Figura 1 - Sistematização da Resource Advantage Theory

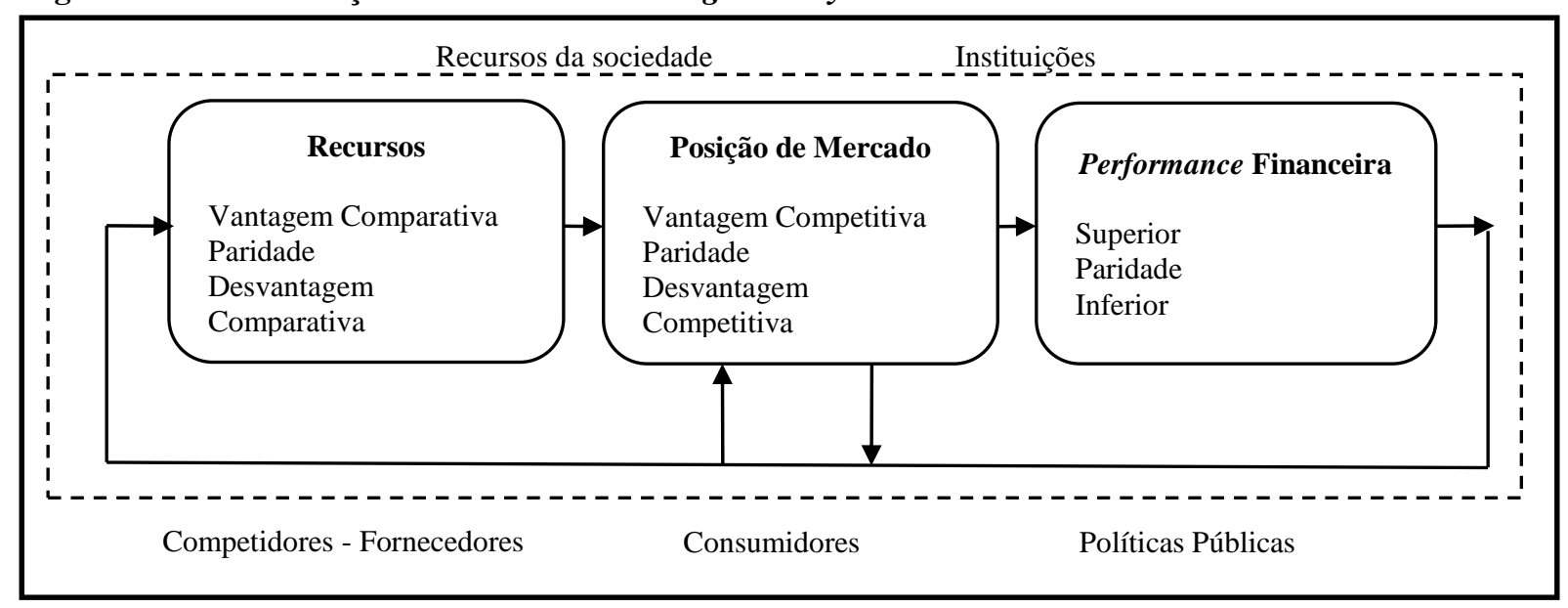

Fonte: Hunt (1997).

Conforme a Figura 1 a vantagem comparativa de recursos é responsável pelas vantagens competitivas de mercado e de desempenho financeiro. No que tange aos recursos, Hunt (2000) salienta que podem ser classificados em tangíveis ou intangíveis a fim de produzir com maior eficiência e/ou eficácia o produto oferecido ao mercado. Conforme Golicic, Fugate e Davis (2012) um desempenho de recursos elevado, indica que as empresas devem superar as suas concorrentes no que tange aos custos relativos, de maneira a alcançar maior eficiência e eficácia.

Em relação ao desempenho de mercado, Priem e Swink (2012) afirmam que os indicadores são oriundos de uma disponibilidade de recursos, sendo que quanto maior o valor percebido pelos clientes, maior seria a vantagem competitiva da empresa, gerando assim uma nova contribuição tanto nos recursos, quanto na performance financeira. Nesse sentido, o 
posicionamento de mercado (vantagem ou desvantagem competitiva) irá impactar na performance financeira (HUNT, 2000). No aspecto de mercado, é importante verificar fatores, como por exemplo, o aumento de produtividade, que incrementam as vendas. Sendo que vendas mais eficazes e eficientes geram mais valor para as empresas.

Em relação a performance financeira, verifica-se um grande avanço na importância atribuída a mensuração do desempenho enquanto ferramenta gerencial nas empresas (KENNERLY, NEELY, 2002; SOUZA, 2011). Conforme Groppelli e Nikbakht (2002), a importância desses indicadores concerne na eficiência da empresa, bem como no controle dos custos e geração de resultados. Conforme Hunt e Morgan (1995) e Rossi e Silva (2009) a posição de vantagem ou desvantagem competitiva resulta em um desempenho financeiro inferior ou superior. Essa vantagem competitiva é alcançada quando os recursos permitem a geração de produtos com um valor superior ou a produção a baixos custos

Na percepção de Hunt e Madhavaram (2012) a RA Theory envolve o processo evolutivo da competição e além disso se utiliza da interdisciplinaridade de outras áreas como marketing, economia, ética, entre outros. Conforme Rossi e Silva (2009) a RA Theory é coerente com os conceitos de aprendizado organizacional, pois com o passar do tempo as empresas aprendem e de diversas maneiras. Essa teoria também amplia o conhecimento do processo de competição. Nesse sentido Hunt (2000) afirma que o feedback desse processo de competição é obtido pelo desempenho financeiro, no qual as empresas reconhecem os recursos e sua posição de mercado.

\subsection{MÉTODO TOPSIS}

O método Technique for Order Preference by Similarity to an Ideal Solution (TOPSIS) proposto por Hwang e Yoon (1981), vem sendo aplicado a pesquisas científicas voltadas ao apoio a decisão multicritério. O método é dado pela matriz de dados $X$, composta por elementos na forma $x_{i j}$, que corresponde ao resultado observado da alternativa $i$ no critério $j$. Assume-se ainda $A=\left\{A_{i} \mid i=1,2, \cdots, n\right\}$ como o conjunto de todas as alternativas e $C=\left\{C_{j} \mid j=\right.$ $1,2, \cdots, m\}$ como conjunto de todos os critérios. Inicialmente realiza-se a padronização dos dados.

Uma das formas de padronização leva em consideração a natureza do critério avaliado. Se o critério representa benefícios (quando maior melhor) a padronização do critério $j$ é dada por: $r_{i j}=\left(x_{i j}-x_{j}^{-}\right) /\left(x_{j}^{+}-x_{j}^{-}\right)$, onde $x_{j}^{-}=\min x_{i j}$ e $x_{j}^{+}=\max x_{i j}$. Se o critério representa custos (quanto menor melhor) a padronização do critério $j$ é dada por: $r_{i j}=\left(x_{j}^{+}-x_{i j}\right) /\left(x_{j}^{+}-\right.$ $\left.x_{j}^{-}\right)$. Esta padronização permite que todos os critérios tenham amplitude 1.

Realizada a padronização, o valor ponderado de cada critério pode ser obtido da seguinte forma $v_{i j}(x)=w_{j} r_{i j}$. Esta ponderação serve para considerar peso maior a critérios que influenciam mais o processo de tomada de decisão. Com base nos valores ponderados, estabelece-se o Cenário Ideal Positivo (CIP) e o Cenário Ideal Negativo (CIN) conforme segue:

$$
\begin{gathered}
C I P=A^{+}=\left\{v_{1}^{+}(x), v_{2}^{+}(x), \cdots, v_{m}^{+}(x)\right\} \\
C I P=A^{+}=\left\{\left(\max _{i} x_{i j} \mid j \in J_{1}\right) \text { ou }\left(\min _{i} x_{i j} \mid j \in J_{2}\right), k=1,2, \cdots, n\right\}
\end{gathered}
$$

Assim como:

$$
\begin{gathered}
C I N=A^{-}=\left\{v_{1}^{-}(x), v_{2}^{-}(x), \cdots, v_{m}^{-}(x)\right\} \\
C I N=A^{-}=\left\{\left(\min _{i} x_{i j} \mid j \in J_{1}\right) \text { ou }\left(\max _{i} x_{i j} \mid j \in J_{2}\right), k=1,2, \cdots, n\right\}
\end{gathered}
$$

Onde $J_{1}$ representa o conjunto de critérios do tipo quanto maior melhor e $J_{2}$ o conjunto de critérios do tipo quanto menor melhor.

O passo seguinte do modelo é calcular as distâncias de cada alternativa para o cenário ideal positivo $\left(D_{i}^{+}\right)$e as distâncias de cada alternativa para o cenário ideal negativo $\left(D_{i}^{-}\right)$. Cabe destacar que o modelo de distâncias pode variar de acordo com o objetivo do problema. Podese utilizar, por simplicidade a distância Euclidiana, assim como as distâncias de Manhattan, Minkowski, Mahalanobis, entre outras. Bastando para isso considerar que o modelo utilizado seja uma métrica definida sobre um espaço métrico. Por simplicidade, será dada sequência 
considerando a distância Euclidiana. Assim o modelo para obtenção das mesmas é definido como:

De igual modo:

$$
D_{i}^{+}=\sqrt{\sum_{j=1}^{m}\left[v_{i j}(x)-v_{j}^{+}(x)\right]^{2}}
$$

Onde $i=1,2, \cdots, n$.

$$
D_{i}^{-}=\sqrt{\sum_{j=1}^{m}\left[v_{i j}(x)-v_{j}^{-}(x)\right]^{2}}
$$

Por fim o método deve sintetizar um score que caracterize o desempenho segundo a similaridade da alternativa avaliada para com o Cenário Ideal Positivo. Este índice pode ser definido como:

$$
C_{i}^{*}=\frac{D_{i}^{-}}{D_{i}^{*}+D_{i}^{-}}
$$

Onde $C_{i}^{*} \in[1,0]$ para todo $i=1,2, \cdots, n$.

O ranqueamento é feito considerando o score obtido no índice de similaridade. Quanto maior o valor, melhor a alternativa foi avaliada em seu desempenho segundo seus critérios.

O modelo descrito por Hwang e Yoon (1981) recomenda a utilização de variáveis numéricas. Alguns trabalhos como Cables, Garcí-Cascales e Lamata (2012) descrevem um modelo Fuzzy para o método TOPSIS que pode utilizar variáveis linguísticas ou categóricas segundo um determinado critério. Possivelmente a aplicação de variáveis categóricas pode ser possibilitada também pelo uso de outra métricas na mensuração das distâncias. A ainda problemas quanto ao estabelecimento de pesos para cada critério de avaliação. Uma alternativa apresenta por Li et al. (2011) é a utilização do modelo de Entropia da Informação, para se estabelecer os pesos $w_{j}$.

Outro modelo que pode ser descrito dentro deste conjunto de métodos é o método VIKOR que será apresentado a seguir.

\subsection{MÉTOdO VIKOR}

O método VlseKriterijumska Optimizacija I Kompromisno Resenje (VIKOR) foi apresentado por Opricovic (1998) que baseou sua ideia em problemas de Programação por Compromisso de Yu (1973) e Zeleny (1982). A ideia inicial do modelo é estabelecer um ranking segundo as distâncias com relação a um cenário ideal. A diferença deste método para o TOPSIS está justamente ligada a vinculação deste método as funções de compromisso. Opricovic e Tzeng (2004) discutem sobre as diferenças entre o método TOPSIS e o método VIKOR. Segundo os autores o método VIKOR tem como virtude, sobre o método TOPSIS ponderar a importância das distâncias em relação aos cenários Ideal e melhor desempenho verificado. Ainda segundo os autores, este comparativo estabelece o método VIKOR como um método onde o ranking formado é mais estável do que o formado pelo método TOPSIS.

O método baseia-se na função do Método de Programação de Compromisso descrita por Yu (1973) e Zeleny (1982). Assumindo como notação tem-se o conjunto de alternativas $A$ definido como $a_{1}, \cdots, a_{n}$, onde para a alternativa $\mathrm{i}$, o valor padronizado de sua avaliação no critério $j$ é dado por $f_{i j}$. Assim pode-se definir a função de compromisso como:

$$
L_{p, i}=\left\{\sum_{j=1}^{m}\left[\frac{w_{j}\left(f_{j}^{*}-f_{i j}\right)}{\left(f_{j}^{*}-f_{j}^{-}\right)}\right]^{p}\right\}^{\frac{1}{p}}
$$


Onde: $1 \leq p \leq \infty$ e que $i=1,2, \cdots, n$, assumindo que $j$ denota um critério específico e que $m$ é o número de critérios utilizado no modelo. Ainda, $f_{j}^{*}$ trata-se do melhor resultado obtido no critério $j$ e que $f_{j}^{-}$trata-se do pior resultado obtido para o critério $j$.

Inicialmente o método VIKOR constrói dois scores utilizados para o ranqueamento, sendo eles $S$ e $R$. A construção destes scores está vinculada a função de compromisso, onde para se construir $S$ assume-se $p=1$ e para a construção de $R$ assume-se $p=\infty$. Desta maneira é possível obter:

E ainda:

$$
S_{i}=\sum_{j=1}^{m}\left[\frac{w_{j}\left(f_{j}^{*}-f_{i j}\right)}{\left(f_{j}^{*}-f_{j}^{-}\right)}\right]
$$

$$
R_{i}=\max _{j}\left[w_{i}\left(f_{j}^{*}-f_{i j}\right) /\left(f_{j}^{*}-f_{j}^{-}\right)\right]
$$

Existe portanto uma curva estabelecida por $F^{C}$ que apresenta o conjunto de soluções viáveis que se destacam como o mais próximo possível do cenário ideal positivo $F^{*}$. A Figura 2 destaca esta representação.

Figura 2 - Solução Ideal e Solução Compromisso

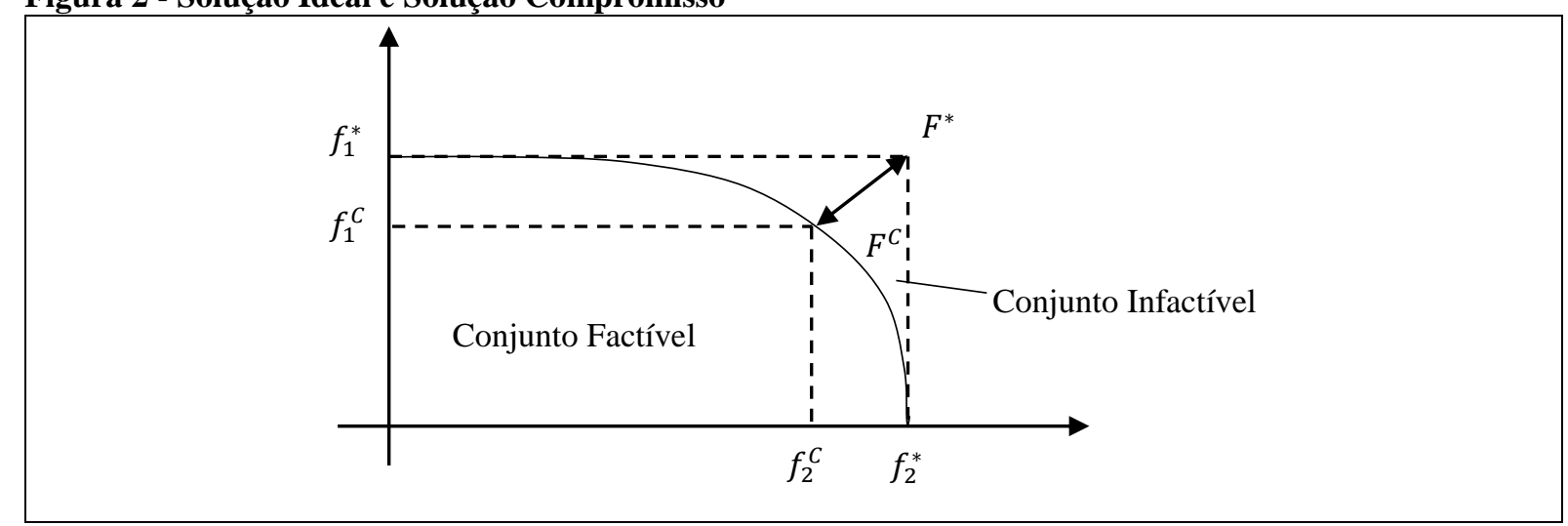

Fonte: Opricovic e Tzeng (2004). etapas:

O método VIKOR de apoio a decisão, pode ser descrito de acordo com as seguintes

Etapa 1: identificação dos valores $f_{j}^{*}=\max _{i} f_{i j}$ e $f_{j}^{-}=\min _{i} f_{i j}$, cabe destacar que é considerado máximo o valor que representa o melhor resultado obtido pela variável, sendo que podem existir variáveis descritas como "quanto maior melhor" e variáveis descritas como "quanto menor melhor". Interpretação análoga é possível para o pior cenário.

Etapa 2: Calcular os valores $S_{i}$ e $R_{i}$ para todo $i=1,2, \cdots, n$. As fórmulas foram descritas anteriormente. Cabe destacar que $w_{j}$ representa o peso estabelecido para a variável. equação:

Etapa 3: Calcular os valores de $Q_{i}$, dado $i=1,2, \cdots, n$. Considerando para tanto a

$$
Q_{i}=v\left(S_{i}-S^{*}\right) /\left(S^{-}-S^{*}\right)+(1-v)\left(R_{i}-R^{*}\right) /\left(R^{-}-R^{*}\right)
$$

Onde $S^{*}=\max _{i} S_{i} ; S^{-}=\min _{i} S_{i}$ assim como $R^{*}=\max _{i} R_{i} ; R^{-}=\min _{i} R_{i}$. O peso $v$ é uma ponderação descrita pelos autores como critério majoritário. Costuma-se defini-lo como $v=0,5$, porém este critério pode sofrer alterações devido a hipótese de robustez da classificação.

Ainda de acordo com Opricovic e Tzeng (2004), a classificação das alternativas segue uma descrição mais difusa do que métodos como TOPSIS. O VIKOR fornece três diferentes rankings, a saber $S, R$ e $Q$, sendo os dois primeiros com preferência a valores maiores e o último com preferência de scores menores. Assim uma alternativa $a^{\prime}$ será classificada como preferida se possuir fechamento com as seguintes condições: 
Condição 1: $a^{\prime}$ possui "Vantagem Aceitável" sobre as demais se quando comparada com $a^{\prime \prime}$ (alternativa imediatamente posterior) for verificado que: $Q\left(a^{\prime \prime}\right)-Q\left(a^{\prime}\right) \geq D Q$ e $D Q=1 /(n-1)$.

Condição 2: $a^{\prime}$ tem "Estabilidade aceitável na tomada de decisão" se além de melhor em $Q$, também for melhor em $S$ e/ou $R$. Esta condição é mais comum em analises onde $v \approx$ 0,5 .

A natureza da comparação final dos rankings dá ao VIKOR um laço possível com outros métodos, principalmente com os modelos de outrankings. Nesta discussão Opricovic e Tzeng (2007) estudaram a extensão do método utilizando argumentos dos métodos PROMETHEE e ELECTRE. Assim os autores concluíram que os resultados obtidos pelo VIKOR são mais similares aos rankings obtidos pelos métodos PROMETEE e ELECTRE II, do que pelo método TOPSIS.

\section{METODOLOGIA}

Conforme delimitação do objetivo da pesquisa, busca-se analisar os indicadores referentes a recursos, mercado e indicadores financeiros, conforme a Resource Advantage Theory que busca explicar a vantagem competitiva das empresas. Para tanto, realizou-se uma pesquisa descritiva, de cunho documental e com uma abordagem quantitativa.

\subsection{PopulaÇão e Amostra}

A população e amostra da pesquisa, compreende as empresas do setor têxtil com ações negociada na BM\&FBovespa. Limita-se a análise da pesquisa para este setor devido a interligação teórica com o mesmo. Pois conforme Slack (1993) este setor atende da melhor maneira a dimensão de mercado que é proposto pela Resource Advantage Theory, relacionando com a vantagem competitiva das empresas.

A população da pesquisa engloba as empresas pertencentes ao setor de Consumo Cíclico e subsetor de Tecidos, Vestuário e Calçados da BMF\&Bovespa, perfazendo um total de 40 empresas. Porém verificou-se que nem todas as empresas apresentavam as informações necessárias para o cálculo dos indicadores requeridos. Para tanto a amostra da pesquisa é composta pelas empresas apresentadas no Quadro 1.

Quadro 1 - Amostra da pesquisa
\begin{tabular}{|l|l|}
\hline Segmento & Empresa \\
\hline Fios e Tecidos & $\begin{array}{l}\text { Buettner, Cedro, Coteminas, Cremer, Ind Cataguas, Karsten, Pettenati, } \\
\text { Santanense, Schlosser, Springs, Teka, Tex Renaux, Wembley }\end{array}$ \\
\hline Vestuário & Cia Hering \\
\hline Calçados & Alpargatas, Cambuci, Grendene, Vulcabras \\
\hline Comércio de Tecidos, Vestuário e Calçados & Arezzo Co, Guararapes, Le Lis Blanc \\
\hline
\end{tabular}

Fonte: dados da pesquisa.

O subsetor de Tecidos, Vestuários e Calçados possui quatro segmentos. O segmento de Fios e Tecidos possui o maior número de empresas (61,90\%), na sequência o segmento de Calçados com 19,05 \% das empresas, o segmento de Comércio com 14,29\%, e o segmento de Vestuário representado com apenas uma empresa.

\subsection{Coleta dOS DAdOS}

Para atender ao objetivo da pesquisa, elaborou-se um constructo para delimitar os indicadores a serem utilizados, pautando-se na Resource Advantage Theory. No Quadro 2, apresenta-se estes indicadores.

Quadro 2 - Indicadores utilizados na pesquisa

\begin{tabular}{|l|l|}
\hline Dimensão & Indicadores \\
\hline
\end{tabular}




\begin{tabular}{|l|l|}
\hline Recursos & Giro de Estoques, Imobilizado, Estoques e Patrimônio Líquido \\
\hline Mercado & Vendas Líquidas/ Ativo Total, Crescimento das vendas e Lucro Líquido \\
\hline Financeira & Retorno sobre Patrimônio (ROE), Liquidez Geral, Liquidez Corrente e Endividamento \\
\hline
\end{tabular}

Fonte: elaborado com base nos autores, Hunt (2000) e Hu e Ansell (2007).

Os indicadores apresentados do Quadro 2, foram coletados na base de dados Economática ${ }^{\circledR}$. Salienta-se que no constructo teórico tem-se um grupo maior de indicadores. Porém restringiu-se a análise devido ao acesso das informações.

\subsection{PROCEDIMENTOS DE ANÁLISE dOS DADOS}

Estabelecidos os indicadores a serem utilizados, o procedimento seguinte pauta-se na definição de rankings para explicar o posicionamento das empresas nas dimensões da Resource Advantage Theory. Além dos modelos de ranqueamento, TOPSIS e VIKOR, utilizou-se ainda neste estudo a correlação de Kendall para variáveis ordinais. A estipulação dos pesos para os cálculos dos modelos de ranqueamento foi feita através do coeficiente de variação de cada uma das variáveis utilizadas.

\section{RESULTADOS DA PESQUISA}

A análise dos dados se dá em duas partes, a primeiro por meio da mensuração do desempenho das empresas têxteis utilizado os outrora descritos métodos de apoio a tomada de decisão. Em um segundo momento será comparado o posicionamento da empresas nos três rankings preconizados pela RAT, a saber: Recursos, Mercado e Finanças. Os resultados são apresentados na sequência.

Tabela 1 - Desempenho no fator Recursos

\begin{tabular}{|c|c|c|c|c|c|c|c|c|}
\hline \multirow{2}{*}{ Nome } & \multicolumn{4}{|c|}{ TOPSIS } & \multicolumn{4}{|c|}{ VIKOR } \\
\hline & $D^{+}(i)$ & $D^{-}(i)$ & $D(i)$ & Ranking & $S(i)$ & $R(i)$ & $Q(i)$ & Ranking \\
\hline Alpargatas & 0,3296 & 0,1904 & 0,3662 & 6 & 0,6057 & 0,2573 & 0,4841 & 5 \\
\hline Arezzo Co & 0,3070 & 0,2395 & 0,4382 & 4 & 0,5465 & 0,2159 & 0,6454 & 4 \\
\hline Buettner & 0,4086 & 0,2423 & 0,3723 & 5 & 0,6007 & 0,3798 & 0,2248 & 13 \\
\hline Cambuci & 0,3877 & 0,1559 & 0,2868 & 17 & 0,6724 & 0,3358 & 0,2335 & 11 \\
\hline Cedro & 0,3944 & 0,1981 & 0,3343 & 9 & 0,6072 & 0,3738 & 0,2300 & 12 \\
\hline Cia Hering & 0,3666 & 0,1823 & 0,3321 & 10 & 0,6205 & 0,3080 & 0,3564 & 7 \\
\hline Coteminas & 0,3625 & 0,1718 & 0,3215 & 11 & 0,6268 & 0,3157 & 0,3322 & 8 \\
\hline Cremer & 0,2178 & 0,4048 & 0,6502 & 1 & 0,3711 & 0,1807 & 0,9332 & 1 \\
\hline Grendene & 0,4178 & 0,1664 & 0,2848 & 18 & 0,6989 & 0,3554 & 0,1591 & 17 \\
\hline Guararapes & 0,4087 & 0,1460 & 0,2631 & 20 & 0,7007 & 0,3616 & 0,1435 & 18 \\
\hline Ind Cataguas & 0,4075 & 0,1791 & 0,3054 & 14 & 0,6522 & 0,3675 & 0,1892 & 15 \\
\hline Karsten & 0,2397 & 0,2662 & 0,5262 & 2 & 0,4871 & 0,1595 & 0,8393 & 2 \\
\hline Le Lis Blanc & 0,2512 & 0,2757 & 0,5232 & 3 & 0,5089 & 0,1499 & 0,8337 & 3 \\
\hline Pettenati & 0,3939 & 0,2043 & 0,3416 & 8 & 0,5926 & 0,3768 & 0,2410 & 10 \\
\hline Santanense & 0,4107 & 0,1829 & 0,3082 & 13 & 0,6410 & 0,3807 & 0,1741 & 16 \\
\hline Schlosser & 0,4495 & 0,1964 & 0,3041 & 16 & 0,7827 & 0,3804 & 0,0037 & 20 \\
\hline Springs & 0,3409 & 0,1948 & 0,3636 & 7 & 0,5786 & 0,2960 & 0,4330 & 6 \\
\hline Teka & 0,4372 & 0,1407 & 0,2435 & 21 & 0,7852 & 0,3792 & 0,0033 & 21 \\
\hline Tex Renaux & 0,4126 & 0,1804 & 0,3042 & 15 & 0,7064 & 0,3592 & 0,1417 & 19 \\
\hline Vulcabras & 0,3951 & 0,1558 & 0,2829 & 19 & 0,6784 & 0,3440 & 0,2085 & 14 \\
\hline Wembley & 0,3658 & 0,1641 & 0,3097 & 12 & 0,6427 & 0,3172 & 0,3096 & 9 \\
\hline
\end{tabular}

Fonte: resultados da pesquisa.

Verifica-se na Tabela 1 que as empresas com melhor posicionamento em ambos os rankings foram, Cremer, Karsten e Le Lis Blanc. O ranqueamento para as primeiras empresas analisadas foi coincidente, havendo pouca variabilidade do posicionamento das demais. A pior empresa, segundo o ranking de desempenho foi a Teka, que apresenta limitação em seu Patrimônio Líquido e dificuldades de captação de recursos. 
Tabela 2 - Desempenho no fator Mercado

\begin{tabular}{l|c|c|c|c|c|c|c|c}
\hline \multirow{2}{*}{ Nome } & \multicolumn{9}{|c}{ TOPSIS } & \multicolumn{4}{c}{ VIKOR } \\
\cline { 2 - 9 } & $D^{+}(i)$ & $D^{-}(i)$ & $D(i)$ & Ranking & $S(i)$ & $R(i)$ & $Q(i)$ & Ranking \\
\hline Alpargatas & 0,1945 & 0,3163 & 0,6192 & 5 & 0,3699 & 0,1317 & 0,7059 & 5 \\
\hline Arezzo Co & 0,1215 & 0,4002 & 0,7671 & 2 & 0,2065 & 0,0874 & 0,9042 & 3 \\
\hline Buettner & 0,4458 & 0,0727 & 0,1402 & 20 & 0,8754 & 0,2527 & 0,1291 & 19 \\
\hline Cambuci & 0,2727 & 0,2518 & 0,4800 & 8 & 0,4998 & 0,1901 & 0,4955 & 8 \\
\hline Cedro & 0,3008 & 0,2196 & 0,4220 & 10 & 0,5733 & 0,1896 & 0,4530 & 11 \\
\hline Cia Hering & 0,0829 & 0,4676 & 0,8494 & 1 & 0,0923 & 0,0823 & 0,9838 & 1 \\
\hline Coteminas & 0,3106 & 0,2013 & 0,3933 & 14 & 0,5992 & 0,1926 & 0,4307 & 13 \\
\hline Cremer & 0,2677 & 0,2449 & 0,4777 & 9 & 0,5165 & 0,1601 & 0,5539 & 6 \\
\hline Grendene & 0,1247 & 0,3883 & 0,7569 & 3 & 0,2433 & 0,0752 & 0,9100 & 2 \\
\hline Guararapes & 0,1751 & 0,3545 & 0,6694 & 4 & 0,3117 & 0,1263 & 0,7528 & 4 \\
\hline Ind Cataguas & 0,3038 & 0,2108 & 0,4097 & 12 & 0,5891 & 0,1838 & 0,4566 & 10 \\
\hline Karsten & 0,3656 & 0,1786 & 0,3282 & 16 & 0,6700 & 0,2484 & 0,2614 & 16 \\
\hline Le Lis Blanc & 0,2784 & 0,3027 & 0,5209 & 7 & 0,4587 & 0,2067 & 0,4823 & 9 \\
\hline Pettenati & 0,3138 & 0,2283 & 0,4211 & 11 & 0,5734 & 0,2150 & 0,3950 & 15 \\
\hline Santanense & 0,2496 & 0,2780 & 0,5269 & 6 & 0,4675 & 0,1802 & 0,5373 & 7 \\
\hline Schlosser & 0,4447 & 0,1658 & 0,2716 & 18 & 0,8287 & 0,2934 & 0,0646 & 20 \\
\hline Springs & 0,3177 & 0,1983 & 0,3842 & 15 & 0,6052 & 0,2023 & 0,4051 & 14 \\
\hline Teka & 0,4769 & 0,0546 & 0,1027 & 21 & 0,9309 & 0,2950 & 0,0000 & 21 \\
\hline Tex Renaux & 0,4116 & 0,1462 & 0,2621 & 19 & 0,7616 & 0,2756 & 0,1451 & 18 \\
\hline Vulcabras & 0,3878 & 0,1650 & 0,2985 & 17 & 0,7082 & 0,2476 & 0,2406 & 17 \\
\hline Wembley & 0,3083 & 0,2031 & 0,3972 & 13 & 0,5962 & 0,1909 & 0,4364 & 12 \\
\hline Fon & & & & & & &
\end{tabular}

Fonte: resultados da pesquisa.

No que tange ao desempenho de mercado descrito na Tabela 2, verifica-se a alternância das empresas Arezzo e Grendene, permanecendo a empresa Cia. Hering como líder do desempenho na dimensão de Mercado. Destaca-se na Tabela 2 que a liderança de Recursos por parte de algumas empresas como Cremer, não implicou na liderança quando considerado o mercado, o que não confirma e partes a teoria de Hunt, Morgan (1996) e Hunt (2000). Verificase na última dimensão a efetividade da teoria quanto a dimensão de desempenho financeiro.

Tabela 3 - Desempenho no fator de performance financeira

\begin{tabular}{l|c|c|c|c|c|c|c|c}
\hline \multirow{2}{*}{ Nome } & \multicolumn{9}{c}{ TOPSIS } & \multicolumn{5}{c}{ VIKOR } \\
\cline { 2 - 9 } & $D^{+}(i)$ & $D^{-}(i)$ & $D(i)$ & Ranking & $S(i)$ & $R(i)$ & $Q(i)$ & Ranking \\
\hline Alpargatas & 0,4061 & 0,1581 & 0,2802 & 9 & 0,6975 & 0,2683 & 0,6143 & 3 \\
\hline Arezzo Co & 0,3520 & 0,2560 & 0,4211 & 2 & 0,5649 & 0,2913 & 0,5708 & 6 \\
\hline Buettner & 0,4479 & 0,1714 & 0,2767 & 11 & 0,7519 & 0,3156 & 0,2240 & 17 \\
\hline Cambuci & 0,4193 & 0,1469 & 0,2595 & 13 & 0,7179 & 0,2909 & 0,4331 & 14 \\
\hline Cedro & 0,4379 & 0,1218 & 0,2177 & 19 & 0,7588 & 0,2691 & 0,5521 & 8 \\
\hline Cia Hering & 0,3741 & 0,2130 & 0,3628 & 6 & 0,6249 & 0,2872 & 0,5447 & 9 \\
\hline Coteminas & 0,4249 & 0,1355 & 0,2418 & 14 & 0,7345 & 0,2658 & 0,5982 & 5 \\
\hline Cremer & 0,4267 & 0,1319 & 0,2361 & 15 & 0,7376 & 0,2758 & 0,5234 & 11 \\
\hline Grendene & 0,3089 & 0,4434 & 0,5894 & 1 & 0,3119 & 0,3089 & 0,6769 & 1 \\
\hline Guararapes & 0,4082 & 0,1582 & 0,2794 & 10 & 0,7006 & 0,2744 & 0,5678 & 7 \\
\hline Ind Cataguas & 0,3976 & 0,1751 & 0,3057 & 7 & 0,6744 & 0,2681 & 0,6372 & 2 \\
\hline Karsten & 0,4304 & 0,1281 & 0,2293 & 17 & 0,7980 & 0,2977 & 0,3108 & 15 \\
\hline Le Lis Blanc & 0,4126 & 0,1523 & 0,2696 & 12 & 0,7053 & 0,2908 & 0,4451 & 13 \\
\hline Pettenati & 0,4325 & 0,1265 & 0,2263 & 18 & 0,7480 & 0,2769 & 0,5056 & 12 \\
\hline Santanense & 0,4008 & 0,1718 & 0,3000 & 8 & 0,6838 & 0,2817 & 0,5308 & 10 \\
\hline Schlosser & 0,4344 & 0,2577 & 0,3723 & 4 & 0,6718 & 0,3234 & 0,2415 & 16 \\
\hline Springs & 0,4270 & 0,1318 & 0,2358 & 16 & 0,7389 & 0,2639 & 0,6077 & 4 \\
\hline Teka & 0,4363 & 0,2544 & 0,3683 & 5 & 0,6757 & 0,3314 & 0,1806 & 19 \\
\hline Tex Renaux & 0,4375 & 0,3169 & 0,4201 & 3 & 0,6200 & 0,3335 & 0,2170 & 18 \\
\hline Vulcabras & 0,4654 & 0,1221 & 0,2078 & 20 & 0,7979 & 0,3165 & 0,1757 & 20 \\
\hline Wembley & 0,4959 & 0,0884 & 0,1513 & 21 & 0,8562 & 0,3212 & 0,0879 & 21 \\
\hline Fonte: resultas & & & & & &
\end{tabular}

Fonte: resultados da pesquisa. 
Os resultados da Tabela 3 destacam o desempenho mensurado para a dimensão de desempenho financeiro. Nesta dimensão a liderança ficou com a empresa Grendene em ambos os rankings, mensurados por meio dos métodos TOPSIS e VIKOR. Quanto as segundas e terceiras posições ocorreram modificações significativas, assim como nas demais. Entretanto, destaca-se que Vulcabras e Wembley foram as duas piores empresas por ambos os rankings.

Desta maneira a RA Theory não se configura consistente, pois nessa pesquisa não podese assumir que uma empresa com vantagem em recursos terá vantagem no mercado e será superior no desempenho financeiro. O Quadro 3 foi formado para a investigação da classificação descrita pelos autores, considerou-se a classificação em três grupos com a mesma quantidade de empresas.

Quadro 3 - Classificação pela RA Theory

\begin{tabular}{|c|c|c|c|c|c|c|}
\hline \multirow{2}{*}{ Nome } & \multicolumn{3}{|c|}{ TOPSIS } & \multicolumn{3}{|c|}{ VIKOR } \\
\hline & Recursos & Mercado & Finanças & Recursos & Mercado & Finanças \\
\hline Alpargatas & Vantagem & Vantagem & Paridade & Vantagem & Vantagem & Superior \\
\hline Arezzo Co & Vantagem & Vantagem & Superior & Vantagem & Vantagem & Superior \\
\hline Buettner & Vantagem & Desvantagem & Paridade & Paridade & Desvantagem & Inferior \\
\hline Cambuci & Desvantagem & Paridade & Paridade & Paridade & Paridade & Paridade \\
\hline Cedro & Paridade & Paridade & Inferior & Paridade & Paridade & Paridade \\
\hline Cia Hering & Paridade & Vantagem & Superior & Vantagem & Vantagem & Paridade \\
\hline Coteminas & Paridade & Paridade & Paridade & Paridade & Paridade & Superior \\
\hline Cremer & Vantagem & Paridade & Inferior & Vantagem & Vantagem & Paridade \\
\hline Grendene & Desvantagem & Vantagem & Superior & Desvantagem & Vantagem & Superior \\
\hline Guararapes & Desvantagem & Vantagem & Paridade & Desvantagem & Vantagem & Superior \\
\hline Ind Cataguas & Paridade & Paridade & Superior & Desvantagem & Paridade & Superior \\
\hline Karsten & Vantagem & Desvantagem & Inferior & Vantagem & Desvantagem & Inferior \\
\hline Le Lis Blanc & Vantagem & Vantagem & Paridade & Vantagem & Paridade & Paridade \\
\hline Pettenati & Paridade & Paridade & Inferior & Paridade & Desvantagem & Paridade \\
\hline Santanense & Paridade & Vantagem & Paridade & Desvantagem & Vantagem & Paridade \\
\hline Schlosser & Desvantagem & Desvantagem & Superior & Desvantagem & Desvantagem & Inferior \\
\hline Springs & Vantagem & Desvantagem & Inferior & Vantagem & Paridade & Superior \\
\hline Teka & Desvantagem & Desvantagem & Superior & Desvantagem & Desvantagem & Inferior \\
\hline Tex Renaux & Desvantagem & Desvantagem & Superior & Desvantagem & Desvantagem & Inferior \\
\hline Vulcabras & Desvantagem & Desvantagem & Inferior & Paridade & Desvantagem & Inferior \\
\hline Wembley & Paridade & Paridade & Inferior & Paridade & Paridade & Inferior \\
\hline
\end{tabular}

Fonte: resultados da pesquisa.

Os resultados do Quadro 3, destacam que apenas a empresa Arezzo foi classificada como com vantagem Competitiva em recursos e mercado e como superior em desempenho financeiro, para ambos os métodos de mensuração do desempenho. A empresa Alpargatas confirma a teoria de Hunt e Morgan (1996) e Hunt (2000) apenas para o desempenho mensurado pelo método TOPSIS. Estes resultados indicam para um problema de verificação da RA Theory no setor têxtil brasileiro. Para confirmação estatística e posterior conclusão, foi feita a análise da Correlação Ordinal de Kendall, entre todos os rankings.

Tabela 4 - Correlação de Kendall entre os rankings de desempenho para cada fator.

\begin{tabular}{|c|c|c|c|c|}
\hline \multicolumn{2}{|c|}{ TOPSIS } & Recursos & Mercado & Finanças \\
\hline \multirow{2}{*}{ Recursos } & Correlation Coefficient & 1,000 & 0,086 & $-0,190$ \\
\hline & Sig. (2-caldas) & - & 0,587 & 0,227 \\
\hline \multirow{2}{*}{ Mercado } & Correlation Coefficient & 0,086 & 1,000 & 0,210 \\
\hline & Sig. (2- caldas) & 0,587 & - & 0,184 \\
\hline \multirow{2}{*}{ Finanças } & Correlation Coefficient & $-0,190$ & 0,210 & 1,000 \\
\hline & Sig. (2- caldas) & 0,227 & 0,184 & - \\
\hline \multicolumn{2}{|c|}{ VIKOR } & Recursos & Mercado & Finanças \\
\hline \multirow{2}{*}{ Recursos } & Correlation Coefficient & 1,000 & 0,229 & 0,133 \\
\hline & Sig. (2- caldas) & - & 0,147 & 0,398 \\
\hline
\end{tabular}




\begin{tabular}{c|c|c|c|c}
\hline \multirow{2}{*}{ Mercado } & Correlation Coefficient & 0,229 & 1,000 & 0,467 \\
\cline { 2 - 4 } & Sig. (2- caldas) & 0,147 & - & 0,003 \\
\hline \multirow{2}{*}{ Finanças } & Correlation Coefficient & 0,133 & 0,467 & 1,000 \\
\cline { 2 - 5 } & Sig. (2- caldas) & 0,398 & 0,003 & - \\
\hline
\end{tabular}

Fonte: resultados da pesquisa.

Os resultados apresentados na Tabela 4, referentes a análise da correlação de Kendall entre as variáveis, constatou a não existência de relação para praticamente a totalidade de comparações. Existe entretanto uma correlação significativa entre os rankings de mercado e financeiro com desempenho mensurado pelo método VIKOR. Mesmo assim os resultados não apontam para a verificação da RA Theory na análise do desempenho de empresas têxteis.

\section{CONCLUSÃO}

Esta pesquisa teve por objetivo analisar o desempenho em recursos, mercado e financeiro que explicam a vantagem competitiva empresarial de acordo com a Resource Advantage Theory, de empresas do setor têxtil listadas na BM\&FBovespa. Foram analisadas um total de 21 empresas do setor têxtil divididas em quatro segmentos. Para a mensuração do desempenho foram adotados os métodos de apoio a decisão multicritério baseados em cenários ideais TOPSIS e VIKOR. Foram analisados os desempenhos com base em quatro indicadores para recursos, três para mercado e quatro para a dimensão financeira.

Os resultados da pesquisa apontam para a não verificação da RA Theory em empresas têxteis, considerando o que se preconiza de que uma empresa em vantagem em recursos e mercado tende a ser superior na dimensão financeira. Desta forma a única empresa que verificou este fator foi a Arezzo. De mesmo modo para verificação da referida teoria seria coerente que existisse correlação entre os rankings das diferentes dimensões, o que não se verificou.

Assim conclui-se que a vantagem competitiva em recursos e em mercado de empresas do setor têxtil do Brasil não contribui para superioridade no desempenho financeiro das empresas.

\section{REFERÊNCIAS}

[1] CABleS, E.; GARCÍA-CASCALES, M. S.; LAMATA, M. T. The LTOPSIS: An alternative to TOPSIS decision-making approach for linguistic variables. Expert Systems with Applications, v. 39, n. 2, p. 2119-2126, 2012.

[2] GOLICIC, S. L.; FUGATE, B. S.; DAVIS, D. F. Examining Market Information and Brand Equity Through Resource-Advantage Theory: A Carrier Perspective. Journal of Business Logistics, v. 33, p. 20-33, 2012.

[3] GROPPELLI, A. A.; NIKBAKHT, E. Administração financeira. São Paulo: Saraiva, 2. ed. 2002.

[4] HU, Y. C.; ANSELL, J. Measuring retail company performance using credit scoring techniques. European Journal of Operational Research, v. 183, p. 1595-1606, 2007.

[5] HUNT, S. D. Resource-advantage theory: an evolutionary theory of competitive behavior. Journal of Economics Issues, v. 31, n. 1, p. 59, 77, 1997.

[6] HUNT, S. D. A General Theory of Competition: Resources, Competences, Productivity, Economic Growth. Thousand Oaks, CA: Sage Publications. 2000.

[7] HUNT, S. D.; MADHAVARAM, S. Managerial action and resource-advantage theory: conceptual frameworks emanating from a positive theory of competition. Journal of Business \& Industrial Marketing, v. 27, n. 7, p. 582-591. 2012.

[8] HUNT, S.D.; MORGAN, R. M. The comparative advantage theory of competition. Journal of Marketing, v. 59, n. 2, p. 1-14. 1995. 
[9] HUNT, S. D.; MORGAN, R. M. The resource-advantage theory of competition: dynamics, path dependencies, and evolutionary dimensions. Journal of Marketing, $\mathrm{v}$. 60, n. 4, p. 107-114. 1996.

[10] HWANG, C. L.; YOON, K. Multiple attribute decision making, methods and applications. Lecture notes in economics and mathematical sýstems, v. 186. New York: Springer-Verlang, 1981.

[11] KENNERLEY, M.; NEELY, A. A framework of the factors affecting the evolution of performance measurement systems. International journal of operations \& production management, vol. 22, n. 11, p. 1222-1245, 2002.

[12] LI, X.; WANG, K.; LIU, L.; XIN, J.; YANG, H.; GAO, C. Application of the entropy weight and TOPSIS method in safety evaluation of coal mines. Procedia Engineering, v. 26, p. 2085-2091, 2011.

[13] LI, X.; WANG, K.; LIU, L.; XIN, J.; YANG, H.; GAO, C. Application of the entropy weight and TOPSIS method in safety evaluation of coal mines. Procedia Engineering, v. 26, p. 2085-2091, 2011.

[14] OPRICOVIC, S. Multicriteria Optimization in Civil Engineering. Faculty of Civil Engineering, Belgrade, 1998.

[15] OPRICOVIC, S.; TZENG, G.-H. Compromise solution by MCDM methods: A comparative analysis of VIKOR and TOPSIS. European Journal of Operational Research, v. 156, n. 2, p. 445-455, 2004.

[16] PRIEM, R. L.; SWINK, M. A demand-side perspective on supply chain management. Journal of Supply Chain Management, v. 48, n. 2, p. 7-13. 2012

[17] ROSSI, R. M.; SILVA, A. L. Vantagem competitiva: operacionalizando o conceito a partir da Resource-Advantage Theory. Revista Ibero-Americana de Estratégia RIAE, São Paulo, v. 8, n. 2, p. 31-57, jul./dez. 2009. DOI: http://dx.doi.org/10.5585/riae.v8i2.1632

[18] SLACK, N. Vantagem competitiva em manufatura: atingindo competitividade nas operações industriais. São Paulo: Atlas, 1993.

[19] SOUZA, A. E. Indicadores de mensuração de desempenho em pequenas e médias empresas (PMEs): Estudo no setor calçadista de Santa Catarina. Tese (Doutorado em Administração) - USP, 2011.

[20] YU, P. L. A class of solutions for group decision problems. Management Science, v. 19, n. 8, p. 936-946, 1973.

[21] ZELENY, M. Multiple Criteria Decision Making. McGraw-Hill, New York, 1982. 